ISSN: $2641-1644$

\title{
Selective Androgen Receptor Modulators (SARMs): A Mini-Review
}

\author{
Michael AB Naafs* \\ Department of Endocrinology, International Health Consultancy, Netherlands
}

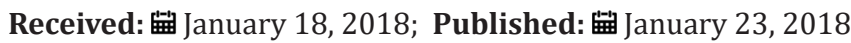

*Corresponding author: Michael AB Naafs, Dutch Internist Endocrinologist, Health Consultant at Naafs, International Health Consultancy, Rhodoslaan 20, 7577KN, Oldenzaal, The Netherlands

\begin{abstract}
Selective Androgen Receptor Modulators (SARMs) were discovered in the late 1990's. They may have an application in treatments of various diseases, including muscle wasting, cancer cachexia, breast cancer, osteoporosis, andropause and sarcopenia. In this minireview the development, pharmacodynamics, and the phase 1 and 2 trial results of the SARMs are discussed with a special emphasis on the illicit use of the SARMs.
\end{abstract}

\section{Introduction}

The androgen receptor (AR) is a member of the steroid hormone receptor family that plays important roles in the physiology and pathology of diverse tissues.AR ligands,which include circulating testosterone and locally synthesized dihydrotestosterone bind to activate the AR to elicit their effects.Ubiquitous expression of the AR metabolism and cross reactivity with other receptors limit broad therapeutic utilisation of steroidal androgens.However,the discovery of selective androgen receptor modulators (SARMs) provide an opportunity to promote the benificial effects with greatly unwanted side effects. In the last two decades SARMs have been proposed as treatments of choice for various diseases, including muscle wasting, breast cancer and osteoporosis. In addition, they may have an application in andropause, sarcopenia, cancer cachexia and as selective anabolic steroids in performing body building sports [1-6]. In this mini-review the development, pharmacodynamics and the phase 1 an 2 trial results of the SARMs are discussed,with a special emphasis on the illicit use of the SARMs.

\section{Development of SARMs}

Synthesized steroidal androgens due to their ability to mimic the actions of their endogenous counterparts have been used ckinically as valuable therapeutic agents to target a variety of male and female disorders resulting from androgen deficiency. The principle clinical indication of androgens is as replacement therapy for hypogonadal men [1,2]. Other documented clinical uses of androgens include delayed puberty in boys, anemias, primary osteoporosis, heriditary angioneurotic edema, endometriosis, estrogen receptor-positive breast cancer and muscular diseases, as Duchenne's muscular dystrophy [3-6].

Since the discovery of the therapeutic benefits of testosterone in the 1930's a variety of androgen preparations have been introduced and tested clinically.

Unfortunately, all current available androgen preparations have severe limitations $[2,6]$. Unmodified testosterone is impractical for oral administration due to its low systematic bioavalability [7]. Testosterone esters (e.g., testosterone propionate and testosterone enanthate) are presently the most widely used testosterone preparations, usually administered by intramuscular injection in oil-vehicles [8,9]. A prolonged duration of action is achieved with these esters. However,they produce highly variable testosterone levels. 17-alpha alkylated testosterones (e.g., methylteststerone and oxandrolone) can be given orally. Nevertheless, they often cause unacceptable hepatotoxicity and are less efficacious; hence they are not recommended for long-term androgen therapy [9-11].

At the end of the 1990's studies with affinity ligands for the androgen receptor started. The discovery of these nonsteoidal androgens offered an opportunity for the development of a new generation of selective androgen receptor modulators 
(SARMs) superior to current androgens. Theoretically, SARMs are advantegeous over their steroidal counterparts in that they can obtain better receptor selectivity and allow greater flexibility in structural modification. Thus SARMs can potentially avoid the undesirable side effects caused by cross-reactivity and achieve superior pharmacokinetic properties [12]

\section{Pharmacodynamics of SARMs}

Structural modifications of the acryl propionamide analogues bicalutamide and hydroxyflutamide led to the discovery of the first generation SARMs. The compounds S1 and S4 in this series bind AR with high affinity and demonstrate tissue selectivity in the Herzberger assay,that utilizes a castrated rat model [13-20]. Both S1 and S4 prevented castration induced atrophy of the levator ani muscle and acted as weak agonists in the prostate. At a dose of $3 \mathrm{mg} / \mathrm{kg} /$ day, S4 partially restored the prostate weight to $<20 \%$ of intact weight, but fully restored the levator ani weight, skeletal muscle strength, bone mineral density, bone strength and lean body mass and suppressed LH(luteinizing hormone) and FSH( follicle stimulating hormone) $[20,21]$.

S4 also prevented ovariectomy-induced bone loss in a female rat model of osteoporosis [22]. The ability of SARMs to promote both muscle strength and bone mechanical strength constitutes a unique advantage over other therapies for osteoporosis, that only increase bone density. S1 and S4 are partial agonists thus in intact male rats [20,21]. S1 and S4 compete with endogenous androgens and act as antogonists in prostate, such SARMs with antagonistic or low intrinsic activity in prostate might be useful in the treatment of benign prostate hyperplasia (BPH) or prostate cancer. The suppressive effects of this class of SARMS on gonadotrofin secretion in rats suggests a potential application for male contraception [21]. The ether linkage and B-ring para-position substitution are critical for agonist activity of the acryl propionamide SARMs [19]. Based on crystal structures, compounds with ether linkage appear to adapt a more compact confirmation than bicalutamide due to formation of an intramolecular H-bond, allowing the B-ring to avoid steric conflict with the side chain of W741 in AR and potentially explaining the agonist activity [23].

The hydantoin derivatives developed by the BMS group have an A-ring structure that is similar to that of bicalutamide. The cyanonitro group of these molecules interact with Q711 and R752 [2426]. The benzene ring or the naphtidyl group,together with the hydantoin ring overlaps the steroid plane, while the hydantoin rings forms a H-bond with N705.BMS-564929 binds AR with high affinity and high specificity. BMS-564929 demonstrated anabolic activity in the levator ani muscle and a high degree of tissue selectivity as indicated by a substantially higher ED50 (Effective Dose for 50\% of the population receiving the drug) for the prostate. Hydantoin derivatives are potent suppressors of LH. BMS $=564929$ is orally available in humans with a half-life of 8-14 hours. The prolonged half-life of these ligands in rats may explain the lower dose needed to achieve pharmacological effects. Differences regarding in vivo activities of SARMs, that share similar binding affinity and in vitro activity,may be related to the differences in pharmacokinetics and drug exposure [27].

Hanada et al. [28] Pharmaceutical Co. reported a series of tetrahydroquinolinone derivatives as AR agonists for bone. Although these compounds displayed high AR affinity and strong agonist activity in prostate and levator ani,they demonstrated little selectivity between androgenic and anabolic tissues [27]. Significant in vivo pharmacological activity was only observed at high subcutaneous doses [27,28]. Ligand Pharmaceuticals developed LGD 2226 and LGD 2941, that are bicyclic 6 anilino quinolinone derivatives, showing anabolic activity on the levator ani muscle as well as on bone mass and strength, while having little effect on prostate size in a preclinical rodent model [29-31]. LGD 2226 was also shown to maintain male reproductive behavior in the castrated rodent model [30].

Scientists at Johnson and Johnson replaced the propionamide linker with cyclic elements such as the pyrazoles, benzimidazoles, indoles and cyclic propionanilide mimetics [31]. Merck scientists have developed a number of 4-azosteroidal derivatives and butanamides [32]. All the above mentioned SARMs belong to the so called "first generation SARMs". The mechanisms that contribute to the tissue specific transcriptional activation and selectivity of biologic effects of the SARMs remain poorly understood.Three general hypotheses have been proposed, although these hypotheses are not mutually exclusive.

a) The coactivator hypothesis assumes that the repertoire of coregulator proteins that associate with the SARM-bound AR differs from that with testosterone-bound AR leading to transcriptional activation of a differentially regulated set of genes.

b) The conformational hypothesis states that functional differences in ligand classes (agonist, antagonists and SARMs) are reflected into conformationally distinct states with distinct thermodynamic partitioning. Ligand binding induces specific conformational changes in the ligand binding domain, which could modulate surface topology and subsequent proteinprotein interactions between the AR and other coregulators involved in genomic transcriptional activation or cytosolic proteins involved in non-genomic signalling. Differences in ligand-specific receptor conformation and protein-protein interactions could result in tissue-specific gene regulation, due to potential changes in interactions with the AR effectors, coregulators or transcriptional factors.

c) The third hypothesis states that the tissue selectivity of SARMs could also be related to differences in their tissue distribution,potential interactions with 5-alpha reductase or 
CYP19 aromatase or tissue specific expression of coregulators [33]. Testosterone actions in some androgenic tissues are amplified by its conversion to 5 -alpha dihydro testosterone [34]. Nonsteroidal SARMS do not serve as a substrate for 5-alpha reductase. Tissue selectivity of SARMs might be related to tissue specific expression of coregulatory proteins. Similarly, some differences of the action of SARM of testosterone could be related to the inability of nonsteroidal SARMs to undergo aromatization.

\section{Preclinical and early clinical trials with SARMs}

A large number of candidate SARMs have undergone preclinical proof of concept and toxicology studies and have made it iinto phase 1 and phase 2 clinical trials [29,35]. These compounds are being positioned for early efficacy trials for osteoporosis,frailty,cancer cachexia and aging-associated fundamental limitations. The use of SARMS for the treatment of androgen defiency in men has been proposed. However, the relative advantages of SARMs over testosterone for this indication are not readily apparant. Many biological features of testosterone, especially its effects on libido and behavior, bone and plasma lipids require its aromatization to estrogen. Because the currently SARMs are neither aromatized nor 5-alpha reduced, these compuonds would face an uphill regulatory bar for FDA approval, as they would be required to show efficacy and safety in many more domains of androgen action, than has been required of testosterone formulations.

While the FDA regulatory pathway for the approval of drugs for osteoporosis has been well delineated, because of precedence set by previously approved drugs, the pathway for approval of function promoting anabolic therapies has not been clearly established. Efforts are underway to generate a consensus around indications, efficacy outcomes in pivotal trials, and minimal clinically important differences in key effective outcomes These efforts should facilitate efficacy trials of candidate molecules. There are 2 types of administering SARMs: orally or in injectable dosages. Well known SARMs are LGD-4033, Ostarine (MK-2866), S4(Andarine), RAD 140, Cardarine(GW 501516) and SR9009. The last two preparations are usually grouped with SARMs, but are not the same and are used as endurance supplements.SARMs have been prohibited by the World Anti-Doping Agency (WADA) since 2008. SARMs have the potential to be misused for performance enhancement in sport due to their anabolic properties, as well as their ability to stimulate androgen receptors in muscle and bone. THey are currently prohibited atall times-in the category of "other anabolic agents" under section S1,2 of the WADA Prohibited List [36] Full clinical FDA approval for human consumption as prescription drugs has not yet been accomplished for any of the SARMs until now.

\section{Ligandrol (LGD-4033)}

Ligandrol is a SARM discovered by Ligand Pharmaceuticals and currently under licensed development by Viking THerapeutics
[37]. There has been a lot of research into the efficacy of SARMs, but very little published research to date on LGD-4033. Ligandrol has exhibited desirable in vivo efficacy on skeletal muscle and bone measurements in animal models of disease. There is only one published study on the effects of LGD-4033 in humans, as well as phase B1 clinical trial results. A 2010 phase 1 clinical trial was the first study in humans of LGD-4033 and evaluated the safety, tolerability and oharmacokinetic profiles of the molecule in a single escalating dose, double-blind, placebo-controlled study in 48 healthy volunteers [38].

In 2013, Bhasia et al. [36] conducted a rigorous 3-week pacebocontrolled study of 76 healthy men (21-50 years),that looked at the safety and tolerability of LGD-4033. During this study participants were randomized to placebo,0,1.0,3 or 1mg LGD-4033 for 21 days. The study evaluated the safety, tolerability, pharmacokinetics and the effects of ascending doses of LGD-4033 on lean body mass, muscle strength, stair climbing power and sex hormones [39]. The sample size was still small and the study was not based on considerations of effect sizes, as the study's primary aim was to establish safety and tolerability, rather than efficacy. Similarly, the 3-week study duration was not designed to demonstrate maximal effects on muscle mass and strength. Therefore larger and longer studies are needed to access the efficacy of LGD-4033. Furthermore the study was supported by Ligand Pharmaceuticals, who developed LGD-4033.

Ligandrol showed a dose-dependent suppression of total testosterone from baseline to 21 days, rather than an increase. Ligandrol did not result in fat loss in this study. It promoted muscle growth, but the evidence is very early weak evidence at this stage. There was an increase in lean body mass, that was doserelated. The mechanisms by which androgens increase muscle mass remain incompletely understood. However, the increase in strength measured by stair climbing speed and power also showed improvement, but not enough to be statistically significant. With a larger sample size and or longer study, it is possible that this effect may be demonstrated. LGD-4033 displayed an immediate effect on hormones in the body from the time it was taken. The research showed gains in lean muscle mass within the 21 days of the study. Adverse effects were not noted. LGD-4033 displayed a prolonged elimination half- life of 24-36 hours. Upon discontinuation of LGD4033 the hormone levels returned to baseline by day 56 [39]. There is just not enough research to show the efficacy of Ligandrol at this stage,despite it was safe and well tolerated at all doses administered.

\section{Ostarine (MK-2866,Enobosarm)}

Merck presented the results of a phase 2 clinical trial evaluating Ostarine (MK-2866),an investigational SARM in patients with cancer induced muscle loss,also known as cancer cachexia at the Endocrine Society Annual Meeting in Washington in 2009 [40]. In this study 159 cancer patients with non-small cell lung cancer, 
colorectal cancer, non-Hodgkins lymphoma, chronic lymphocytic leukemia or breast cancer were randomized. Participants received placebo,1mg or $3 \mathrm{mg}$ Ostarine daily for 16 weeks. Average weight loss prior to entry was 8,8 percent and patients were allowed to receive standard chemotherapy during the trial. The drop-out rate during the trial was $33 \%$.

Ostarine treatment led to statistically significant increases in lean body mass (LBM) and improvement in muscle performance measured by stair climbing in patients with cancer cachexia compared to baseline in both the Ostarine $1 \mathrm{mg}$ and $3 \mathrm{mg}$ cohorts. In the study Ostarine met the primary endpoint of LBM mesured by DEXA(dual energy x-ray absorptiometry) scan, by demonstrating significant increases in LBM compared to baseline in both the Ostarine $1 \mathrm{mg}$ and $3 \mathrm{mg}$ treatment was $0,1 \mathrm{~kg}(\mathrm{p}=0,874$ compared to baseline, $1,5 \mathrm{~kg}(\mathrm{p}=0,001)$ and $1,3 \mathrm{~kg}(\mathrm{p}=0,0045)$ at the end of the 16 week trial.

The study also met the secondary endpoint of muscle function as measured by a 12 step stair climbing test measuring speed and calculating power with each Ostarine treatment arm demonstrating a statistically significant average decrease in time to completion and average percentage increase in power exerted.The change from baseline in stair climb power in the placebo,1mg,and $3 \mathrm{mg}$ treatment groups was 0,23 Watts ( $p=0,66$,compared to baseline)8,4Watts $(p=0,002)$ and 10,1 Watts ( $p=0,001)$,respectively. A critical appraisal results in the same critics,as depicted for the Ligandrol results. Ostarine is also known as Enobosarm and S-22 SARM by various licensing contracts in the body building world.

Ostarine had already shown significant improvement in the ability of healthy,elderly men and women to climb stairs in a phase2A study in 2007.Elderly men and women improved climbing stairs in speed and power,accompanied by significant increases in LBM and decreases in fat mass after only 86 days [41]. Enobasarm (GTx-024,0starine and S-22) is the most well characterized clinically and has consistently demonstrated increases in LBM and better physical function across several populations, along with a lower hazard ratio for survival in cancer patients. Enobosarm was eveluated in the POWER 1(Prevention and Treatment of Muscle Wasting in Patients with Cancer)and in the POWER 2 trial. These are the first phase 3 trials for a SARM. Full results from these studies will soon be published and will guide the development of future anabolic trials [42].

\section{Andarine (S4)}

Andarine(S4) was studied in 120 ovariectomized rats for 120 days. The study found that treatment with S4 (Andarine) was benificial to maintain cortical bone content and whole body and trabecular bone mineral density (BMD) measured by DEXA scan. The S4 treatment also decreased body fat and increased body strength in these animals. It was further disclosed by this study that S4 had the ability to reduce the incidence of fractures via minimizing the incidence of falls, through increased muscle strength and through direct effects on bone,as compared to current therapies that are primarily antiresorptive in nature. The study also found that dosages of S4 were effective to increase LBM and reduce body fat in intact and ovariectomized rats. It was also revealed that Andarine provides the unique potential to prevent bone resorption, increase skeletal muscle mass and strength positions and promotes bone anabolism, that makes it a possible new alternative for the treatment of osteoporosis [43]. To date there are no clinical human studies of Andarine in osteoporosis.Andarine has a half-life of 4-6 hours and is prized for weight loss and building and repair of muscle as a muscle boosting supplement in the fitness community.

\section{RAD 140 (Teslolone)}

RAD 140 is a SARM that stimulates muscle weight increases at a lower dose than that required to stimulate prostate weight. It results in the expected lowering of lipids (LDL,HDL,triglycerides), without elevation of liver enzyne transaminase levels.RAD 140 has excellent pharmacokinetic properties and is a potent anabolic [44]. RAD 140 is a potent AR agonist in breast cancer cells with a distinct mechanism of action,including the AR-mediated repression of estrogen receptor1 (ESR1).It inhibits the growth of multiple AR/ ER+ breast cancer PDX (patient -derived xenograft) models as a single agent,and in combination with palbociclib.These preclinical data present support for further investigation of RAD 140 in AR/ ER+ breast cancer patients [45].

In addition, RAD 140 demonstrated initial preclinical efficacy of a SARM in neuroprotective actions relevant to ALzheiner's disease and related neurodegenerative disease. In cultured hippocampal neurons, RAD 140 was as effective as testosterone in reducing cell death by apoptotic insults. RAD 140 neuroprotection was dependent on MAPK (mitogen-activated-protein) kinase signalling, as evidenced by elevation of ERK (extracellular-signalregulated kinase) phosphorylation and inhibition of protection by the MAPK kinase inhibitor UO 126.Importantly, RAD 140 was also neuroprotective in vivo, using the rat kainate lesion model in experiments with gonadectomized adult rats. RAD 140 was shown to inhibit peripheral tissue specific androgen action, that largely spared prostate, neural efficacy as demonstrated by activation of androgenic gene regulation effects and neuroprotection of hippocampal neurons against cell death caused by systemic administration of the excitotoxin kainate [46]. There are no clinical human studies of RAD 140 until now.

In the fitness community Testolone is seen as one of the latest additions to the line of SARMs. Testolone is developed by Radius Health Company. The increase in LBM and fat loss are highly appreciated, as its androgenic-anabolic ratio of 90:1, compared to testosterone. Recommended dosages of Testolone vary from 20$30 \mathrm{mg}$ once daily and it is used in cycles of 12-14 weeks duration. Because Testolone does not interact with the aromatase enzyme and is not liver toxic, no adverse effects are claimed. The half-life of Testolone is estimated 12-18 hours. 


\section{Cardarine(GW 501516) and SR 9009 (Stenab0olic)}

These two preparations are usually grouped with the SARMs in the fitness community, but are not the same. Cardarine is used as an enhancing running endurance supplement. Cardarine is not a SARM, but a peroxisome proliferative activated receptor-omega agonist (PPAR-omega), that increases PPAR-omega, and regulates muscle metabolism and reprograms muscle fibre types to enhance running training endurance. While training alone increases the exhaustive running performance Cardarine treatment enhances running endurance and the proportion of succinate dehydrogenase(SDH)positive muscle fibres in both trained and untrained mice. It appeared while training increases energy availability by promoting protein catabolism and gluconeogenesis, Cardarine enhances specific consumption of fatty acids and reduces glucose utilisation [47]. In the fitness community Carderine is regarded as"king of the gym". Half-life is between 16-24 hours and it should be taken at 10 mg once a day or twice daily. It is claimed to be useful in conjunction with anabolics and stimulants of any kind without adverse reactions in 12-14 week cycles.

SR 9009 (Stenabolic) is a REV-ERB (revised-viral nuclear erythroblastosis receptors) agonist, that can modulate the expressions of circadian core clock proteins and therefore help to modulate the circadian rythm. Modulation of the REV-ERB activity by synthetic agonists e.g., SR 9009 SR 9011 alters the expression of genes involved in lipid and glucose metabolism and, therefore plays an important role in maintaining the energy homeotasis. Effects of SR9009 and SR9011 in animal studies are increased basal oxygen consumption, decreased lipogenesis, cholesterol and bileacid synthesis in the liver, increased mitochondrial content, glucose and fatty oxidation in the skeletal muscle and decreased lipid storage in the white adipose tissue. The observed increase in energy expenditure and decrease in fat mass make the REV-ERB agonists promising drug candidates for the treatment of several metabolic disorders.They are also attractive for performance enhancement by athletes. Such use can be classified as doping [48].

SR9009 (Stenabolic) has been developed by Scripps Research by the team of Prof. Thomas Burris. Stenabolic is taken orally as a metabolism enhancer in the fitness community. It is believed to have results similar to Cardarine, but with considerable more extra benefits. It is recommended as a very good addition to any steroid (Anavar or Trembolone) or SARMs cycle, especially when used together with Cardarine. The half-life is short, 30-60 minutes,so the dose should be spaced through the day e.g.,10 mg 4-6 times daily. Again no adverse effects are reported.

\section{Illicit use of SARMs}

Recently, the FDA issued a consumer warning letter against supplement-like bodybuilding products, that contain SARMs. The FDA warning came on the heels of warning letters sent to three companies, that market products containing the ingredients. FDA had this to say about the offending products distributed by Infantry
Labs LLC,Iron Mag Labs and Panther Sports Nutrition: “ Although the products identified in the warning letters are marketed and labeled as dietary supplements, they are not dietary supplements. The products are unapproved drugs, that have not been reviewed by the FDA for safety and effectiveness" [49]. FDA told consumers among the dangers associated with SARMs are liver toxicity and the potential to increase the risk of heart attack and stroke.But the agency said the long- term effects of these substances are unknown. However, these FDA health risk statements can not be supported by the few small clinical human phase 1 and2 SARMs studies performed and the ongoing POWER trials. Furthermore, the FDA did not mention that Ostarine and Ligandrol have previously been investigated as new drugs, which makes them ineligible for use as dietary supplements.

Nevertheless, as clinical research of SARMs is slow, we are now in the wonderful situation the real world clinical SARMs experience is now represented by the fitness and body building world. It is estimated that ther are between 2 and 4 million young people in the U.S. alone, who have used performance-enhancing drugs sometime in their life. There are thousands of internet sites offering SARMs in and outside the U.S [50]. So the magnitude of the problem is completely unknown, if there is any problem at all. In general,these young people are very concerned about their health and "looks" and have the good right of their own responsability.

A recent JAMA publication found that the chemical analysis of 44 products sold via the internet as SARMs revealed, that only $52 \%$ contained SARMs and another 39\% contained another unapproved drug. In addition, $25 \%$ of products contained substances not listed on the label, 9 percent did not contain an active substance and 59\% contained substance amounts,that differed from the label [50]. Although these figures must be frightening,there is no registered SARMs epidemic at the U.S. emergency rooms. At present the biggest problems are the "loopholes" in the FDA regulation of dietary supplements.

\section{Conclusion}

The SARMs were discovered in the late 1990's. Clinical development is slow. Few human phase 1 and 2 clinical studies are available Results of the phase 3 POWER trials,studying SARMs in wasting, are awaiting and will guide the development of future anabolic trials. Until now no SARM has received FDA approval. Due to "loopholes" in the FDA regulations the SARMs are widespread used as dietary supplements in the fitness community and body building world. This results in the wonderful situation the clinical experience with SARMs is represented by illicit SARMs use and not by clinical science

\section{References}

1. Conway AJ, Boylan LM, Howe C, Ross G, Handelsman DJ (1988) Randomized clinical trial of testosterone replacement therapy in hypogonadal men. Int J Androl 11(4): 247-264. 
2. Wu FC (1992) Testicular steroidgenesis and androgen use and abuse. Baillieres Clin Endocrinol Metab 6(2): 373-403.

3. Bagatell CJ, Bremner WJ (1996) Androgens in men-uses and abuses. N Engl J Med 334(11): 707-714.

4. Nieschlag E (1996) Testosterone replacement therapy: something old, something new. Clin Endocrinol(Oxf) 45(3): 261-262.

5. Bhasin S, Tenover JS (1997) Age-associated sarcopenia-issues in the use of testosterone as an anabolic agent in older men. J Clin Endocrinol Metab 82(6): 1659-1660.

6. Bhasin S, Bremmer WJ (1997) Emerging issues in androgen replacement therapy. J Clin Endocrinol Metab 82(1): 3-8.

7. Handelsman DJ, Conway AJ, Boylan LM (1990) Pharmacokinetics and pharmacodynamics of testosterone pellets in man. J Clin Endocrinol Metab 71(1): 216-222.

8. Snyder PJ, Lawrence DA (1980) Treatment of male hypogonadism with testosterone enathate. J Clin Endocrinol Metab 51(6): 1335-1339.

9. Velazquez E, Bellebarba AG (1998) Testosterone replacement therapy. Arch Androl 41(1): 79-90.

10. Heywood R, Chesterman H, Ball SA, Wadsworth PF (1977) Toxicity of methyltestosterone in the beagle dog. Toxicology 7(3): 357-365.

11. Ishak KG, Zimmerman HJ (1987) Hepatotoxic effects of the anabolic/ androgenic steroids. Semin Liver Dis 7(3): 230-236.

12. Dalton JT, Mukherjee A, Zhu Z, Kirkovsky L, Miller DD (1998) Discovery of nonsteroidal androgens. Biochem Biophys Res Commun 244(1): 1-4.

13. Mukherjee A, Kirskovsky L, Yao XT (1996) Enantiose selective binding of Casodex to the androgen receptor. Xenobiotica 26: 117-122.

14. Mukherjee A, Kirskovsky L, Kimura Y, Marvel MM, Miller DD, et al. (1999) Affinity labeling of the androgen receptor with nonsteroidal chemoaffinity ligands. Biochem Pharmacol 58(8): 1259-1267.

15. Negro-Vilar A (1999) Selective androgen receptor modulators (SARMs): a novel approach to androgen therapy for the new millenium. J Clin Endocrinol Metab 84(10): 3459-1362.

16. Hamman LG, Mani NS, Davis RL (1999) Discovery of a potent,orally active, nonsteroidal androgen receptor agonist 4-ethyl-1-2,3,4,-tetrahydro-6(trifluoro methyl)8-pyridino (5,6-9)-guinoline. J Med Chem 42: 210-212.

17. Naafs Michael AB (2017) Pharmacodynamic evaluation: Endocrinology Chapter 35. In:Drug Discovery and Evaluation Methods in Clinical Pharmacology: (2 ${ }^{\text {nd }}$ edn). Hock FJ, Gralinski MR (Eds.), Springer Verlag Berlin, Heidelberg, New York, USA.

18. Yin D, He Y, Perera MA, Hong SS, Marhefka C, et al. (2003) Key structural features of nonsteroidal ligands for binding and activation of the androgen receptor. Mol Pharmacol 63(1): 211-223.

19. Gao W, Kearby JD, Nair VA, Chung K, Parlow AF, et al. (2004) Comparison of the pharmacological effects of a novel selective androgen receptor modulator, the 5-alpha reductase inhibitor finasteride and the antandrogen hydroxyflutamidein intact rats:new approach for benign prostate hyperplasia. Endocrinology 145(12): 5420-5428.

20. Gao W, Reiser PJ, Coss CC, Phelps MA, Kearbey JD, et al. (2005) Selective Androgen Receptor Modulator (SARM) Treatment Improves Muscle Strength and Body Composition and Prevents Bone Loss in Orchdectomized Rats. Endocrinolgy 146(11): 4887-1497.

21. Gao W, Reiser PJ, Kearby JD (2004) Effects of a Novel Selective Androgen Recepor Modulator (SARM) on Skeletal Muscle Mass and Strength in Castrated Male Rats. The Endocrine Society, New Orleans, USA.
22. Kearby JD, Gao W, Narayam R, Di Wu, Duane DM, et al. (2007) Selective Androgen Receptor Modulator (SARM) treatment prevents bone loss and reduces body fat in ovarictomized rats. Pharmaceutical Research 24(2): 328-335.

23. Bohl CE, Gao W, Miller DD, Bell CE, Dalton JT (2005) Structural basis for antagonism and resistance of bicalutamide in prostate cancer. Proc Natl Acad Sci USA 102(17): 6201-6206.

24. Hamann LG, Manfredi MC, Sun C (2007) Tandem optimization of target activity and elimination of mutagenic potential in a potent series of $\mathrm{N}$-acryl bicyclic hydantoine based selective androgen receptor modulators. Bioorganic\& Medicinal Chemistry Letters 17: 1860-1864.

25. Manfredi MC, Bi Y, Nirschl AR, Ostrowski J, Hamann LG, et al. (2007) Synthesis and SAR of tetrahydropyrrolol $(1,2-b)(1,2,5)$ thiadiazol($2(3 \mathrm{H})$-one-1,1-dioxide analogues as highly potent selective androgen receptor modulators. Bioorganic\&Medicinal Chemistry Letters 17(16): 4487-4490.

26. Ostrowski J, Kuhms JE, Lupisella JA, Manfredi MC, Beehler BC, et al. (2007) Pharmacological and x-ray structural characterization of a novel selective androgen receptor modulator: potent hyperanabolic stimulation of skeletal muscle with hypostimulation of prostate in rats. Endocrinology 148(1): 4-12.

27. Kim J, Wu D, Hwang DJ, Miller DD, Dalton JT (2005) The para substituent of S-3-(phenoxy)-2-hydroxy-2-methyl-N-(4nitro3 -trifluoromethyl-phenyl)prop-ionamides is a major structural determinant of in vivo disposition and activity of selective androgen receptor modulators. J Pharmacol Exp Ther 315(1): 230-239.

28. Hanada K, Furaya K, Yamamoto N, Nejishima H, Ichikawa K, et al. (2003) Bone anabolic effects of S-40503, a novel nonsteroidal selective androgen receptor modulator in rat models of osteoporosis. Biol Pharm Bull 26(11): 1563-1569.

29. Narayanan R, Mohler ML, Bohl CE, Miller DD, Dalton JT (2008) Selective androgen receptor modulators in preclinical and clinical development. Nuclear Receptor Signalling 6: e010.

30. Miner JN, Chang W, Chapman MS, Finn PD, Hong MH, et al. (2007) An orally active selective androgen receptor modulator is efficacious on bone,muscle and sex function with reduced impact on prostate. Endocrinology 148(1): 363-373.

31.van Oeveren A, Motamedi M, Martinborough E, et al. (2007) Novel selective androgen receptor modulators:SAR studies on 6-bisalkylamino2-quinolinones. Bioorganics \& Medicinal Chemistry Letters 17(6): 1527-1531.

32. van Oeveren A, Motamedi M, Manis S, Keith BM, Francisco JL et al. (2006) Discovery of 6-N,N,bis(2,2,2,trifluoroethyl), amino-4trifluoromethylquinolin-2(1H0-one as a novel selective androgen receptor modulator. Journal of Medicinal Chemistry 49(21): 6143-6146.

33. Ng RA, Lanter JC, Alford VC, Allan GF, Sbriscia T, et al. (2007) Synthesis of potent and tissue-selective androgen receptor modulators (SARMs): 2-(2,2,2)-Trifluoroethyl-benzidimazole scaffold. Bioorganic\& Medicinal Chemistry Letters 17(6): 1784-1787.

34. Heinlein CA, Chang C (2002) Androgen receptor (AR) coregulators:an overview. Endocr Rev 23(2): 175-200.

35. Hamman LG (2004) Discovery and preclinical profile of a highly potent and muscle selective androgen receptor modulator (SARM). 27 $7^{\text {th }}$ National Meeting of the American Chemical Society Medicinal Chemistry Division.

36. Bhasia S, Calof OM, Storer TW, Martin L, Norman AM, et al. (2006) Drug insight:Testosterone and selective androgen receptor modulators as anabolic therapies for chronic illness and ageing. Nature Clinical Practice Endocrinology \& Metabolism 2(3): 146-159. 
37. Geyer H, Schanzer W, Thevis M (2014) Anabolic agents: recent strategies for their detection and protection from inadvertent doping. Br J Sports Med 48(10): 820-826.

38. Basaria S, Collins L, Sheffield MM, Dillon EL, Orwoll K, et al. (2011) Safety and Tolerability of LGD-4033, a Novel-Non-Steroidal Selective Androgen Receptor Modulator (SARM) in Healthy Men. The Endocrine Societys 93 ${ }^{\text {rd }}$ Annual Meeting \& Expo June 4-7 Boston, USA.

39. Basaria SI, Collins L, Dillon EL, Orwoll K, Storer TW, et al. (2013) The safety, pharmacokinetics and effects of LGD-4033, a novel nonsteroidal oral selective androgen receptor modulator,in healthy young men. Bio Sci Med Sci 68(1): 87-95.

40. Dalton JT, Taylor RP, Mohler M, Steiner MS (2013) Selective androgen receptor modulators for the prevention and treatment of muscle wasting associated with cancer. Curr Opin Support Palliat Care 7(4): 345-351.

41. Dalton JT (2007) Therapeutic Promise of Selective Androgen Receptor Modulators (SARMs): Preclinical and Clinical Proof-of -Concept Studies. Annual Meeting of the Endocrine Society 41-42.

42. Crawford J, Prado CM, Johnston MA, Richard JG, Ryan PT, et al. (2016) Stydy Design and Rationale for the Phase 3 Clinical DEvelopment Program of Enobosarm, a Selective Androgen Receptor Modulator for the Prevention and Treatment of Muscle Wasting in Cancer Patients (POWER Trials). Curr Oncol Rep 18: 37.

43. Kearby JD, Gao W, Fisher SJ, Wu D, Miller DD, et al. (2009) Effects of selective androgen receptor modulator (SARM) treatment in osteopenic female rats. Pharm Res 26(11): 2471-2477.
44. Miller CP, Shormali M, Lyttle CR, et al. (2011) Design, Synthesis and Preclinical Characterization of the Selective Androgen Receptor RAD 140. ACS Med Chem Lett 2(2): 124-129.

45. Yu Z, He S, Wang D, Patel HK, Miller CP, et al. (2017) Selective Androgen Receptor Modulator RAD 140 Inhibits theGrowth of Androgen/Estrogen Receptor-Positive Breast Cancer Models With a Distinct Mechanism of Action. Clin Cancer Res 23(24): 7608-7620.

46. Javaraman A, Christenson A, Moser VA, Vest RS, Miller CP, et al. (2014) Selective androgen receptor modulator RAD 140 is neuroprotective in cultured neurons and kainate-lesioned male rats. Endocrinology 155(4): 1398-1406.

47. Chen W, Gao R, Xie X, Zheng Z, Li H, et al. (2015) A metabolomic study of the PPARo agonist GW 501516 for enhancing running endurance in Kunming mice. Sci Rep 6(5): 9884.

48. Geldof L, Deventer K, Roels K, Tudela E, Peter VE, et al. (2017) In Vitro Metabolic Studies of REV-ERB Agonists SR9009 and SR9011. FDA USA Food \& Drug Administration. Int J Mol Sci17(10): 1676.

49. Scutti S (2017) Bodybuilding drugs sold online often contained unapproved substances,study says. CNN USA.

50. Van Wagoner RM, Eichner A, Bhasin S, Deuster PA, Eichner D (2017) Chemical Composition and Labeling of Substances Marketed as Selective Androgen Receptor Modulators and Sold via the Internet. JAMA 318(20): 2004-2010.

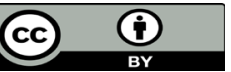

This work is licensed under Creative Commons Attribution 4.0 License

Submission Link:

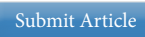

DOI: $10.32474 /$ OAJRSD.2018.01.000103

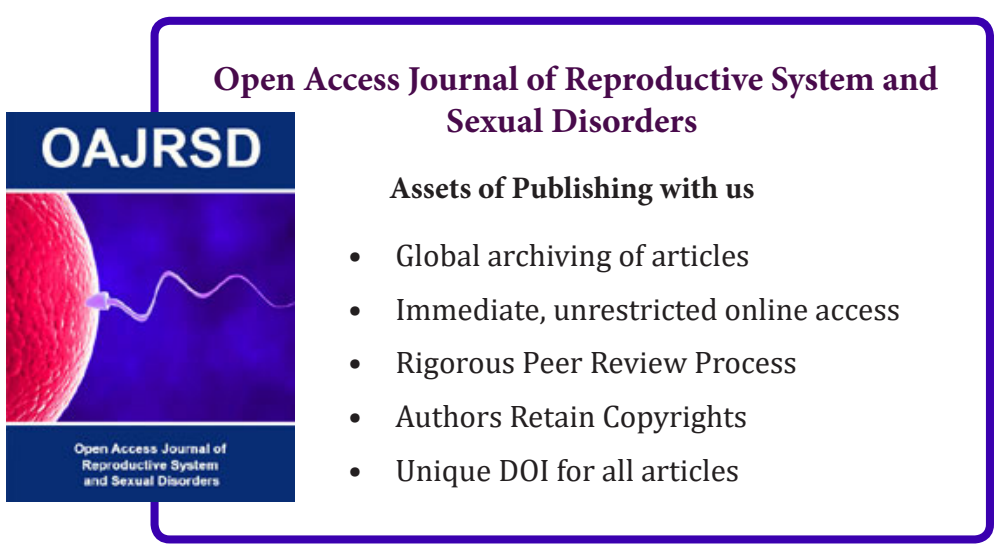

